\title{
UTILIZAÇÃO DE MUDAS DE EUCALYPTUS GRANDIS MELHORA A CRIAÇÃO DO PREDADOR BRONTOCORIS TABIDUS (HET.: PENTATOMIDAE) EM LABORATÓRIO
}

\section{T.V. Zanuncio ${ }^{1}$, C.H.F. Gomides ${ }^{1}$, P.S.R. Vargas ${ }^{1}$, J.M. Souza ${ }^{1}$, F.S. Ramalho ${ }^{2}$, J.E. Serrão ${ }^{1}$, J.C. Zanuncio ${ }^{1}$}

${ }^{1}$ Universidade Federal de Viçosa, Departamento de Biologia Animal/BIOAGRO, Av. Peter Henry Rolfs, s/no, CEP 36571-000, Viçosa, MG, Brasil. E-mail: zanuncio@ufv.br

\section{RESUMO}

Predadores generalistas são importantes para o controle biológico de pragas por se alimentarem de muitas espécies de insetos, especialmente daquelas em maior abundância, e por se manterem em diferentes agroecossistemas, mesmo em períodos de escassez de presa, tendo sido registrados em surtos de Lepidoptera desfolhadores em plantações de eucalipto. Foram estudados a longevidade e os parâmetros reprodutivos do predador Brontocoris tabidus (Signoret) (Heteroptera: Pentatomidae) a $26 \pm 2{ }^{\circ} \mathrm{C}, 65 \pm 10 \%$ de U.R. e fotofase de 12 horas, alimentado com Tenebriomolitor L. (Coleoptera: Tenebrionidae) (T1) ou com esta presa e mudas de Eucalyptus grandis (T2). A longevidade de machos e fêmeas foi de 27,4 $\pm 4,4$ e 39,6 6 3,4 dias e de 27,0 \pm 4,6 e 34,5 $\pm 3,1$ dias, respectivamente. As fêmeas foram sempre mais pesadas que os machos, com 141,1 e 104,9 mg apenas com T. molitor, e 148,8 e 108,3mg com essa presa e mudas de E. grandis, respectivamente. A viabilidade dos ovos, o número de ovos/fêmea e a longevidade de B. tabidus foram maiores no tratamento com essa presa e mudas de E. grandis.

PALAVRAS-CHAVE: Brontocoris tabidus, controle biológico, predador.

\begin{abstract}
USEOF EUCALYPTUSGRANDIS IMPROVESTHERAISINGOFTHEPREDATOR BRONTOCORIS TABIDUS (HET.: PENTATOMIDAE) IN THE LABORATORY.Generalist predators are important for the biological control of pests by feeding on many insect pests, especially those occurring in larger abundance. For this reason, they can maintain their populations in different ecosystems, even during periods of prey shortage, and they are found in outbreaks of Lepidoptera defoliators in eucalyptus plantations. The longevity and reproductive parameters of the predator Brontocoris tabidus(Signoret) (Heteroptera: Pentatomidae) were studied at $26 \pm 2{ }^{\circ} \mathrm{C}, 65 \pm 10 \%$ relative humidity and 12 hours photophase with Tenebrio molitor L. (Coleoptera: Tenebrionidae) (T1) pupae or with this prey and seedlings of Eucalyptus grandis (T2). The longevity of males and females of this predator were 27.4 \pm 4.4 and $39.6 \pm 3.4$ days and $27.0 \pm 4.6$ and $34.5 \pm 3.1$ days, respectively. Females were always heavier than males, with 141.1 and $104.9 \mathrm{mg}$ only with T. molitor and 148.8 and $108.3 \mathrm{mg}$ with this prey and E. grandis seedlings, respectively. The viability and number of eggs/female and the longevity of $B$. tabidus had higher values in the treatment with T. molitor pupae and seedlings of E. grandis.
\end{abstract}

KEY WORDS: Brontocoris tabidus, Eucalyptus grandis, Tenebrio molitor.

\section{INTRODUÇÃO}

O Manejo Integrado de Pragas (MIP) visa reduzir problemas como a resistência de pragas e o impacto dos pesticidas sobre inimigos naturais (ZANUNCIO et al., 1990; GonRING et al., 2003; Lemos et al., 2003). O controle biológico constitui tática importante em programas de MIP pela manipulação de patógenos e artrópodes, incluindo pentatomídeos predadores em áreas reflorestadas (LACERDA et al., 2004), onde as defesas das plantas podem ser benéficas (RUBERSON et al., 1986), maléficas ou não terem efeito (VIVAN et al., 2003) sobre esses inimigos naturais.

A taxa reprodutiva de predadores é maior com abundância de alimento e tende a diminuir à medida que a deficiência alimentar aumenta para beneficiar a sobrevivência, sendo relatado que percevejos predadores melhor alimentados aumentam o ganho de

${ }^{2}$ Empresa Brasileira de Pesquisa Agropecuária, Centro Nacional de Pesquisa de Algodão, Unidade de Controle Biológico, Campina Grande, PB, Brasil. 
peso, a freqüência de ataque às presas, a postura e se desenvolvem mais rapidamente (Oliveira et al., 2004).

Verificou-se que Supputius cincticeps (Stal) (Heteroptera: Pentatomidae) tevemenor períodoninfal e fêmeas mais pesadas quando foi alimentado com pupas de T. molitor e sobre mudas de Eucalyptus urophylla S.T.Blake (Myrtaceae) (Assis Junior et al., 1998; Zanuncio et al., 2005). Podisus nigrispinus Dallas (Heteroptera: Pentatomidae) teve menor duração dos estádios com Bombyx mori L., 1758 (Lepidoptera: Bombycidae) (FERNANDEs et al., 1996) e resultados semelhantes, exceto no quarto estádio, quando alimentado em plantas de algodoeiro ou tomateiro e presas que, apenas, com presas (Oliveira et al., 2002). Além disso, a disponibilidade do algodoeiro e de plantas daninhas reduziu o impacto da escassez parcial de presas na duração, viabilidade dos estádios e da fase ninfal desse predador, mas ele não alcançou a maturação sexual e não ovipositou sem presa (Evangelista Junior et al., 2003; EvANGELISTA Junior, 2004).

Fêmeas de Podisus connexivus Bergroth $(=P$. nigrispinus) (Heteroptera: Pentatomidae) apresentaram maior ganho de peso com E. urophylla e presa. Podisus maculiventris (Say) (Heteroptera: Pentatomidae) apresentou maior sobrevivência e menores períodos de pré-oviposição e ninfal com presas e folhas de batata (RUBERSON et al., 1986).

A longevidade de $P$. nigrispinus foi semelhante para ambos os sexos, com machos apresentando maior longevidade com presas e plantas de algodoeiro (OLIvEIRA et al., 2002). Além disso, o tipo de alimento não afetou a longevidade de adultos de Podisus sculptus Distant (1889) (Heteroptera: Pentatomidae) com diferença, apenas, entre sexos (NAscimento et al., 1997). Fêmeas de P.maculiventris apresentaram maior sobrevivência com plantas de batata ou de tomateiro que, apenas, comágua até $035^{\circ}$ dia de vida, com valores de 31,9;28,8 e27,1 dias, com plantas de batata, tomateou, apenas, água, respectivamente (VALICENTE; O'NeIL, 1995). A disponibilidade da presa com plantas de algodoeiro aumentou a longevidade de P.nigrispinus, com 30,3 $\pm 10,53$ dias para fêmeas e de 43,8 $\pm 17,70$ para machos, contra $16,0 \pm 19,93$ e $11,4 \pm 4,56$ dias para fêmeas e machos desse predador, respectivamente, apenas, com presa (Oliveiraet al., 2002). A habilidade de predadores em obter umidade e nutrientes de plantas pode ser considerada uma adaptação por melhorar a sobrevivência desses em períodos de escassez de presas (De ClercQ; Degheele, 1992; Coll; GUERSHON, 2002).

O objetivo deste trabalho foi estudar o efeito da associação de mudas de Eucalyptus grandis Hill Ex Maiden (Myrtaceae) e pupas de T. molitor sobre a reprodução e a longevidade de $B$. tabidus.

\section{MATERIAL E MÉTODOS}

A pesquisa foi conduzida no Laboratório de Controle Biológico de Insetos do Instituto de Biotecnologia Aplicada a Agropecuária (BIOAGRO) da Universidade Federal de Viçosa, em Viçosa, MG, a $26 \pm 2^{\circ} \mathrm{C}$, U.R. de $65 \pm 10 \%$ e fotofase de 12 horas.

Os tratamentos foram: $\mathrm{T} 1=B$. tabidus alimentado com pupas de T. molitor, e T2=B. tabidus com pupas de T. molitor e mudas de E. grandis, em delineamento inteiramente casualizado com 14 repetições. Cada parcela teve um casal de $B$. tabidus, em uma garrafa de plástico transparente de dois litros (Assis JunIor et al., 1998), com o fundo cortado para permitir o encaixe de uma tampa decopo plástico $(500 \mathrm{~mL})$ furado nocentro, onde foi inserido um tubete com uma muda de $E$. grandis e fixado à garrafa com fita adesiva. Uma abertura circular com, aproximadamente, $4 \mathrm{~cm}$ de diâmetro foi feita nasua extremidadesuperior, ondefoicolocado um copo com o fundo coberto por organza com as presas. Água destilada foi fornecida aos percevejos por um furo no terço superior do copo, em um tubo plástico tipo anestésico fechado com algodão na extremidade apenas. As tampas dos copos do tratamento com presa tiveram um furo central. As posturas de B. tabidusforam acondicionadas em placas de Petri $(9,0 \mathrm{~cm} \times 1,5 \mathrm{~cm})$ com um chumaço de algodão umedecido com água destilada. Diariamente, pupas predadas de T. molitor eram trocadas e completava-se a água dos tubos, sendo as mudas de E. grandis irrigadas, diariamente, etrocadas a cada três dias.

Os adultos de B. tabidus foram pesados logo após a emergência, sexados de acordo com a genitália externa (Mourão et al., 2003; Lemos et al., 2005) e transferidos para os recipientes do teste. As posturas desse predador foram acondicionadas em placas de Petri $(9,0 \mathrm{~cm} \times 1,5 \mathrm{~cm})$ com algodão umidecido em água destilada. Os parâmetros biológicos: mortalidade e longevidade dos adultos, dia de acasalamento, número de ovos por postura e por fêmea, viabilidade dos ovos e períodos de incubação, pré-oviposição, oviposição e pós-oviposição foram avaliados diariamente. Os dados foram submetidos à análise de variância, sendo os números de posturas, de ovos/ fêmea e de ovos/postura transformados em $\sqrt{x+0,5}$.

\section{RESULTADOS E DISCUSSÃO}

Os períodos de oviposição e pós-oviposição de $B$. tabidus foram de 13,1 $\pm 2,0$ e 16,2 $\pm 2,6$, e 14,6 4 2,2 e 11,9 $\pm 2,1$ dias com presa e presa e planta, respectivamente, valores semelhantes aos desse predador com larvas de Muscadomestica L. (Diptera: Muscidae) (JusselinoFILHO et al., 2003), mas maiores que com B. mori (BARCELOs et al., 1991; FERNANDES et al.,1996). Noentanto, esses parâmetros 
podemserafetados por outrosfatores, poisfêmeas desse predador com peso corporal de 95 a $150 \mathrm{mg}$ e de 160 a $220 \mathrm{mg}$ apresentaram maior período de pré-oviposição $(16,53 \pm 3,33$ e 16,09 \pm 2.57 dias, respectivamente) (OLIVEIRA etal., 2005) que os do presente trabalho. O período de oviposição desse predador foi de 5,7 a 10,4 dias com pupas de T. molitor, $M$. domestica e a combinação de ambas, respectivamente (Jusselino FILHO et al., 2003). O período de oviposição de fêmeas de B. tabidus foi de 61,6 e 35,0 dias com lagartas vivas ou previamente congeladas de B. mori, respectivamente (BARCELOS et al., 1994), o que pode ser devido a melhor qualidade nutricional dessaúltima presa. Noentanto, isso difereentreespécies de predadores, pois o período de oviposição de fêmeas de $S$. cincticeps, pesadas eleves, foi de 25,61 $\pm 6,79$ e 29,10 $\pm 3,56$ dias (OliverRa et al., 2003) e o de Podisus distinctus (Stal)(Heteroptera:Pentatomidae) de 15,00 $\pm 7,40 \mathrm{com} B$. mori; $11,28 \pm 9,40$ com T. molitor e 7,60 $\pm 1,00$ com $M$. domestica (LACERDA et al., 2004). Novamente, os valores elevados para esse períodomostram a melhor qualidade nutricional de $B$. mori como presa para esse predador (Lemoset al., 2003; Lemoset al., 2005).

O período de pós-oviposição de $B$. tabidus foi de 14,6 e 11,9 dias, apenas, com presa e presa e planta, respectivamente (Tabela 1 ), valores semelhantes aos desse predador com lagartas de B. mori(BArcelosetal., 1994). Períodos mais longos de pós-oviposição podem permitir às fêmeas de $B$. tabidus se alimentarem e contribuírem para o sucesso do controle biológico (Assis Junior et al., 1998). O número de posturas e de ovos/postura de B. tabidus com T. molitor ou esta presa e mudas de $E$. grandis foi de 1,9 e 2,3 e de 20,5 e 27,6, respectivamente (Tabela 1$)$.
O número de ovos / fêmea de B. tabidus com presa e planta foi maior que, apenas, com pupas de $T$. molitor, de forma semelhante aos $26,5 \pm 8,10$ ovos/ postura de P. nigrispinus com T. molitor (OliveIRaet al., 2002). Fêmeas mais longevas de predadores podem ovipositar mais vezes e permanecer no campo durante maior período de tempo (Assis Junioret al., 1998), mas podem ser afetadas pelo tipo de alimento, pois fêmeas de P. maculiventris e Podisus sagitta (F.) (Heteroptera: Pentatomidae) (= $P$. nigrispinus) não ovipositaram, quando alimentados, apenas, com vagem de feijão ou folhas de batata, necessitando de presas para reprodução (De Clerce; Degheele, 1992).

O período de incubação de ovos de B. tabidus foi de 5,9 e 5,7 dias com pupas de T. molitor e com essa presa e mudas de E. grandis, respectivamente (Tabela 1), semelhante ao desse predador com larvas de $M$. domestica (Jusselino FilHo et al., 2003), e aos 5,7 e 5,5 dias com lagartas de $B$. mori vivas ou previamente congeladas (BARCELOS et al., 1994), mostrando que essa característica não deve ser afetada pelo tipo de presa ou pela inclusão de planta à dieta com presa desse predador.

A viabilidade dos ovos de B. tabidus foi de $41,3 \%$ e $67,9 \%$ com presa e presa e planta, respectivamente (Tabela 1), inferiores aos $75,4 \%$ para fêmeas de $B$. tabidus com pupas de T. molitor; aos 78,8\% e 77,0\% para esse predador com lagartas vivas ou previamente congeladas de B. mori (BARCELOs et al., 1994) e aos $85,19 \%$ e $79,25 \%$ para P. nigrispinus com Spodoptera frugiperda (J. E. Smith) (Lepidoptera: Noctuidae) e T. molitor, respectivamente (OLIVEIRA et al., 2004).

Tabela 1 - Parâmetros reprodutivos, peso e longevidade deBrontocoris tabidus (Heteroptera: Pentatomidae) alimentado com Tenebrio molitor (Coleoptera: Tenebrionidae) (T1) ou com T. molitor e mudas de Eucalyptus grandis $(\mathrm{T} 2)\left(\mathrm{T}=26 \pm 2^{\circ} \mathrm{C}\right.$; $\mathrm{UR}=60 \pm 10 \%$ e fotofase de 12 horas).

\begin{tabular}{llrr}
\hline & & $\mathrm{T} 1$ & $\mathrm{~T} 2$ \\
\hline & Pré-oviposição & $5,9 \pm 1,3 \mathrm{a}$ & $8,0 \pm 1,3 \mathrm{a}$ \\
Períodos & Oviposição & $13,1 \pm 2,0 \mathrm{a}$ & $16,2 \pm 2,6 \mathrm{a}$ \\
& Pós-oviposição & $14,6 \pm 2,2 \mathrm{a}$ & $11,9 \pm 2,1 \mathrm{a}$ \\
& Incubação & $5,9 \pm 0,1 \mathrm{a}$ & $5,7 \pm 0,2 \mathrm{a}$ \\
Viabilidade dos Ovos (\%) & $41,3 \% \mathrm{a}$ & $67,9 \% \mathrm{~b}$ & $2,3 \pm 0,5 \mathrm{a}$ \\
& Posturas & $1,9 \pm 0,5 \mathrm{a}$ & $97,0 \pm 23,9 \mathrm{~b}$ \\
Números de & Ovos por Fêmea & $67,0 \pm 19,1 \mathrm{a}$ & $27,6 \pm 5,2 \mathrm{~b}$ \\
& Ovos por Postura & $20,5 \pm 5,5 \mathrm{a}$ & $108,3 \pm 3,7 \mathrm{a}$ \\
Intervalo entre Posturas & $5,4 \pm 0,5 \mathrm{a}$ & $6,6 \pm 1,5 \mathrm{a}$ & $148,8 \pm 5,8 \mathrm{a}$ \\
Pesos (mg) & Machos & $104,9 \pm 4,0 \mathrm{a}$ & $39,6 \pm 3,4 \mathrm{~b}$ \\
& Fêmeas & $141,1 \pm 5,4 \mathrm{a}$ & $34,5 \pm 3,1 \mathrm{~b}$ \\
\hline
\end{tabular}

Valores seguidos de mesma letra, na mesma linha, não diferem entre si pelo teste Kruskal-Wallis $(\mathrm{P}<0,05)$ 
O intervalo entre posturas de B. tabidus foi de 5,4 e 6,6 dias com pupas de T.molitor ou essa presa e mudas de E. grandis, respectivamente (Tabela 1). Entretanto, menores valores desse parâmetro são desejáveis por permitir a produção de maior número de ovos em menor período de tempo.

O peso de machos e fêmeas de $B$. tabidus foi de 104,9 e de 141,1mg, apenas, com T. molitor e de 108,3 e $148,8 \mathrm{mg}$ com presa e mudas de E. grandis, respectivamente (Tabela 1). O maior ganho de peso das fêmeas está relacionado à necessidade de adquirirem reservas nutricionais e energia para suprir as exigências de esforços reprodutivos (OliveIRA et al., 2005).

A longevidade de machos e fêmeas de B. tabidusfoi de 27,4 e 27,0 e de 39,6 e 34,5 dias com pupas de $T$. molitor e esta presa e planta, respectivamente (Tabela 1), mostrando a importância de presa e planta para a longevidade desse predador (ZANUNCIO et al., 1997; ZANUNCIO et al., 2001).

Brontocoris tabidus deve ser criado com presas e mudas de eucalipto para se reduzir a mortalidade e aumentar a longevidade e o número de ovos e ninfas produzidos por fêmea desse predador (ZANUNCIOetal., 2000). Isto mostra que $B$. tabidus pode obter nutrientes de presas e de plantas, como relatado para outros predadores (Lemos et al., 2001).

Maiores longevidade de fêmeas e número de ovos de B. tabidus com T. molitor e mudas de E. grandis são importantes por melhorarem o potencial desse predador para o controle biológico. Entretanto, apenas o tipo de presa pode não afetar a longevidade de percevejos predadores, pois machos e fêmeas acasalados de $S$. cincticeps apresentaram longevidade semelhante, de 19,2 $\pm 3,3$ e 21,2 $\pm 2,7$ dias e de $29,2 \pm 6,1$ e 30,7 $\pm 4,1$ dias, quando alimentados com $M$. domestica e $T$. molitor, respectivamente.

\section{CONCLUSÕES}

A associação de E. grandis à dieta de B. tabidus é benéfica para esse predador, pelos aumentos no número de posturas, ovos/fêmea, ovos/postura e viabilidade dos ovos desse predador. Porém, o intervalo entre posturas de B. tabidus aumentou em $22,2 \%$ com presa e planta de eucalipto, talvez devido aos seus compostos. Entretanto, para emprego desse predador no controle biológico em plantações de eucalipto, esse intervalo deve ser menor, aumentando o número de insetos atuando e tornand o o controle mais efetivo no menor tempo.

O aumento do peso e da longevidade de machos e fêmeas de B. tabidusalimentados apenas com T.molitor em planta de eucalipto pode ser devido ao elevado investimento de recursos e energia da fêmea na repro- dução. A adição de plantas pode reduzir a taxa de mortalidade de predadores e contribuir para melhorar a sobrevivência de $B$. tabidus no campo, mesmo em períodos de escassez de presas, e aumentar seu desempenho no controle de insetos-praga de eucalipto.

\section{AGRADECIMENTOS}

Ao Conselho Nacional de Desenvolvimento Científico e Tecnológico (CNPq), à Coordenação de Aperfeiçoamento de Pessoal de Nível Superior (CAPES)e à Fundação de Amparo à Pesquisa do Estado de Minas Gerais (FAPEMIG).

\section{REFERÊNCIAS}

ASSIS JUNIOR, S.B.; ZANUNCIO, T.V.: SANTOS, G.P.; ZANUNCIO, J.C. Efeito da suplementação de folhas de E. urophylla no desenvolvimento e reprodução do predador Supputius cincticeps (Stal) (Heteroptera: Pentatomidae). Anais da Sociedade Entomológica do Brasil, v.27, n.2, p.245-253, 1998.

BARCELOS, J.A.V.; ZANUNCIO, J.C.; SANTOS, G.P.; REIS, F.P. Viabilidade da criação em laboratório de Podisus nigrolimbatus (Spinola, 1852) (Hemiptera: Pentatomidae) sobre duas dietas. Revista Árvore, v.15, n.3, p.316-322, 1991.

BARCELOS, J.A.V.; ZANUNCIO, J.C.; OLIVEIRA, A.C.; NASCIMENTO, E.C. Performance em duas dietas e descrição dos adultos de Brontocoris tabidus (Signoret) (Heteroptera: Pentatomidae). Anais da Sociedade Entomológica do Brasil, v.23, n.3, p.519-524, 1994.

COLL, M.; GUERSHON, M. Omnivory in terrestrial arthropods: mixing plant and prey diets. Annual Review of Entomology, v.47, n.1, p.267-297, 2002.

DE CLERCQ, P.; DEGHEELE, D. Plant feeding by two species of predatory bugs of the genus Podisus (Heteroptera: Pentatomidae). Medelingen van Faculteit Landbouwwetenchappen Universiteit Gent, v.57, n.3, p.591-596, 1992.

EVANGELISTA JUNIOR, W.S.; GONDIM JUNIOR, M.G.C.; TORRES, J.B.; MARQUES, E.J. Efeito de plantas daninhas e do algodoeiro no desenvolvimento, reprodução e preferência para oviposição de Podisus nigrispinus (Dallas) (Heteroptera:

Pentatomidae). Neotropical Entomology, v.32, n.4, p.677684, 2003.

EVANGELISTA JUNIOR, W.S.; GONDIM JUNIOR, M.G.C.; TORRES, J.B.; MARQUES, E.J. Fitofagia de Podisus nigrispinus em algodoeiro e plantas daninhas. Pesquisa Agropecuária Brasileira, v.39, n.5, p.413-420, 2004. 
FERNANDES, L.G.; CARVALHO, C.F.; BUENO, V.H.P.; DINIZ, L.C. Aspectos biológicos de Brontocoris tabidus Signoret, 1852 e Podisus nigrispinus Dallas, 1851 (Hemiptera: Pentatomidae). Cerne, v.2, n.1, p.1-10, 1996.

GONRING, A.H.R.; PICANÇO, M.C.; LEITE, G.L.D.; SUINAGA, F.A.; ZANUNCIO, J.C. Seletividade de inseticidas a Podisus rostralis (Stal) (Heteroptera: Pentatomidae) predador de lagartas desfolhadoras de eucalipto. Revista Árvore, v.27, n.2, p.263-268, 2003.

JUSSELINO FILHO, P.; ZANUNCIO, J.C.; FRAGOSO, D.B.; SERRÃO J.E.; LACERDA, M.C. Biology of Brontocoris tabidus (Heteroptera: Pentatomidae) fed with Musca domestica (Diptera: Muscidae) larvae. Brazilian Journal of Biology, v.63, n.3, p.463-468, 2003.

LACERDA, M.C.; FERREIRA, A.M.R.M.; ZANUNCIO, T.V.; ZANUNCIO, J.C.; BERNARDINO, A.S.; ESPINDULA, M.C. Development and reproduction of Podisus distinctus (Heteroptera: Pentatomidae) fed on larvae of Bombyx mori (Lepidoptera: Bombycidae). Revista Brasileira de Biologia, v.64, n.2, p.237-242, 2004.

LEMOS, W.P.; MEDEIROS, R.S.; RAMALHO, F.S.; ZANUNCIO, J.C. Effects of plant feeding on the development, survival and reproduction of Podisus nigrispinus (Dallas) (Heteroptera: Pentatomidae). International Journal of Pest Management, v.47, n.2, p.8993, 2001.

LEMOS, W.P.; RAMALHO, F.S.; SERRÃO, J.E.; ZANUNCIO, J.C. Effects of diet on development of Podisus nigrispinus (Dallas) (Heteroptera:

Pentatomidae), a predator of the cotton leafworm. Journal of Applied Entomology, v.127, n.7, p.389-395, 2003.

LEMOS, W.P.; ZANUNCIO, J.C.; SERRÃO, J.E. Attack behavior of Podisus rostralis (Heteroptera: Pentatomidae) adults on caterpillars of Bombyx mori (Lepidoptera: Bombycidae). Brazilian Archives of Biology and Technology, v.48, n.6, p.975-981, 2005.

MOURÃO, S.A.; ZANUNCIO, J.C.; MOLINARUGAMA, A.J.; VILELA, E.F.; LACERDA, M.C. Effect of prey shortage on the reproduction and longevity of Supputius cincticeps (Stål) (Heteroptera: Pentatomidae). Neotropical Entomology, v.32, n.3, p.469-473, 2003.

NASCIMENTO, E.C.; ZANUNCIO, J.C.: PICANÇO, M.C.; ZANUNCIO, T.V. Desenvolvimento de Podisus sculptus Distant, 1889 (Heteroptera: Pentatomidae) em Bombyx mori (Lepidoptera: Bombycidae) e Tenebrio molitor (Coleoptera: Tenebrionidae). Revista Brasileira de Biologia, v.57, n.2, p.195-201, 1997.

OLIVEIRA, H.N.; PRATISSOLI, D.; PEDRUZZI, E.P.; ESPINDULA, M.C. Desenvolvimento do predador Podisus nigrispinus alimentado com S. frugiperda, e Tenebrio molitor L. (Coleoptera: Tenebrionidae). Pesquisa Agropecuária Brasileira, v.39, n.10, p.947-951, 2004.
OLIVEIRA, J.E.M.; TORRES, J.B.; MOREIRA, A.F.C.; BARROS, R. Efeito das plantas do algodoeiro e do tomateiro, como complemento alimentar, no desenvolvimento e na reprodução do predador Podisus nigrispinus (Dallas) (Heteroptera: Pentatomidae). Neotropical Entomology, v.3, n.1, p.101-108, 2002.

OLIVEIRA, I.; ZANUNCIO, J.C.; SERRÃO, J.E.; MILAGRES, J.M. Reproductive potential of the predator Supputius cincticeps (Heteroptera: Pentatomidae) affected by female body weight. Acta Scientiarum, v.25, n.1, p.49-53, 2003.

OLIVEIRA, I.; ZANUNCIO, J.C.; SERRÃO, J.E.; ZANUNCIO, T.V.; PINON, T.B.M.; FIALHO, M.C.Q. Effect of female weight on reproductive potential of the predator Brontocoris tabidus (Signoret, 1852) (Heteroptera: Pentatomidae). Brazilian Archives of Biology and Technology, v.48, n.2, p.295-301, 2005.

RUBERSON, J.R.; TAUBER, M.J.; TAUBER, C.A. Plant feeding by Podisus maculiventris (Hemiptera: Pentatomidae): effect on survival, development and preoviposition period. Environmental Entomology, v.15, n.4, p.894-897, 1986.

VALICENTE, F.H.; O'NEIL, R.J. Effects of host plants and feeding regimes on selected life-history characteristics of Podisus maculiventris (Say) (Heteroptera: Pentatomidae). Biological Control, v.5, n.3, p.449-461, 1995.

VIVAN, L.M.; TORRES, J.B.; VEIGA, A.F.S.L. Development and reproduction of a predatory stinkbug, Podisus nigrispinus in relation to two different prey types and environmental conditions. BioControl, v.48, n.2, p.155-168, 2003.

ZANUNCIO, J.C.; FAGUNDES, M.; ANJOS, N.; ZANUNCIO, T.V.; CAPITANI, L.C. Levantamento e flutuação populacional de lepidópteros associados a eucaliptocultura: V-Região de Belo Oriente, MG, junho de 1986 a maio de 1987. Revista Árvore, v.14, n.1, p.3544, 1990.

ZANUNCIO, J.C.; TORRES, J.B.; BERNARDO, D.L.; DE CLERCQ, P. Effects of prey switching on nymphal development of four species of predatory stinkbugs. Medelingen van Faculteit Landbouwwetenchappen Universiteit Gent, v.62, n.2b, p.483-490, 1997.

ZANUNCIO, J.C.; ZANUNCIO, T.V.; GUEDES, R.N.C.; RAMALHO, F.S. Effect of feeding on three Eucalyptus species on the development of Brontocoris tabidus (Het.: Pentatomidae) fed with Tenebrio molitor (Col.:

Tenebrionidae). Biocontrol Science and Technology, v.10, n.4, p.443-450, 2000.

ZANUNCIO, J.C.; MOLINA-RUGAMA, A.J.; SERRÃO, J.E.; PRATISSOLI, D. Nymphal development and reproduction of Podisus nigrispinus (Heteroptera: 
Pentatomidae) fed with combinations of Tenebrio molitor (Coleoptera: Tenebrionidae) pupae and Musca domestica (Diptera: Muscidae) larvae. Biocontrol Science and Technology, v.11, n.3, p.331-337, 2001.

ZANUNCIO, J.C.; BESERRA, E.B.; MOLINA-RUGAMA A.J.; ZANUNCIO, T.V.; PINON, T.B.M.; MAFFIA, V.P. Reproduction and longevity of Supputius cincticeps
(Het.: Pentatomidae) fed with larvae of Zophobas confusa, Tenebrio molitor (Col.: Tenebrionidae) or Musca domestica (Dip.: Muscidae). Brazilian Archives of Biology and Technology, v.48, n.5, p.771-777, 2005.

Recebido em 25/4/07

Aceito em 14/4/08 